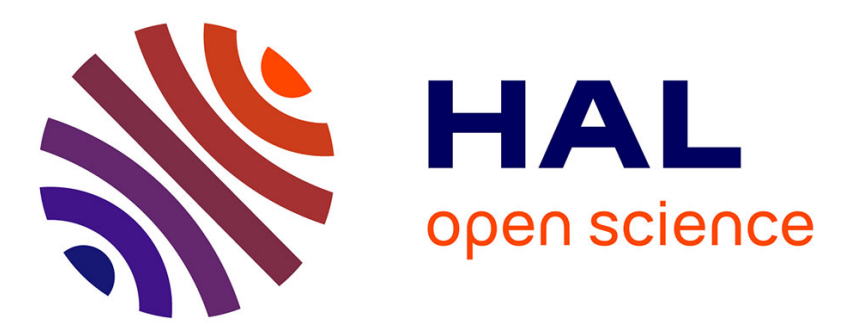

\title{
Energy dependence of double photoionization in $\mathrm{He}$
}

\author{
N. Berrah, F. Heiser, R. Wehlitz, J. Levin, S. Whitfield, J. Viefhaus, I. Sellin, \\ U. Becker
}

\section{To cite this version:}

N. Berrah, F. Heiser, R. Wehlitz, J. Levin, S. Whitfield, et al.. Energy dependence of double photoionization in He. Journal de Physique IV Proceedings, 1993, 03 (C6), pp.C6-197-C6-205. 10.1051/jp4:1993619 . jpa-00251704

\section{HAL Id: jpa-00251704 https://hal.science/jpa-00251704}

Submitted on 1 Jan 1993

HAL is a multi-disciplinary open access archive for the deposit and dissemination of scientific research documents, whether they are published or not. The documents may come from teaching and research institutions in France or abroad, or from public or private research centers.
L'archive ouverte pluridisciplinaire HAL, est destinée au dépôt et à la diffusion de documents scientifiques de niveau recherche, publiés ou non, émanant des établissements d'enseignement et de recherche français ou étrangers, des laboratoires publics ou privés. 


\title{
Energy dependence of double photoionization in He
}

\section{N. BERRAH ${ }^{*, * *}$, F. HEISER*, R. WEHLITZ* , J. LEVIN***, S.B. WHITFIELD*, J. VIEFHAUS*, I.A. SELLIN ${ }^{* * * *}$ and U. BECKER ${ }^{*}$}

${ }^{*}$ Fritz-Haber-Institut der Max-Planck-Gesellschaft, Berlin, Germany

** Western Michigan University, Physics Department, Kalamazoo, MI 49009, U.S.A.

${ }^{* * *}$ National Institute of Standards and Technology, Gaithersburg, MD 20899, U.S.A.

**** University of Tennessee, Physics Department, Knoxville, TN 37996 and Oak Ridge National Laboratory, Oak Ridge, TN 37891, U.S.A.

\begin{abstract}
The ratio of double-to-single ionization of He has been measured between $280 \mathrm{eV}$ and $1210 \mathrm{eV}$ to investigate its energy dependence in the intermediate region. The new intermediate energy measurement, compared with the most recent theories of Pan and Kelly and of Hino and Hino et al, show the importance of including not only ground state but also final state correlations. They also appear to indicate the importance of including higher-order effects in the theory. The previously reported $[11,22]$ high energy measurements between $2-12 \mathrm{keV}$ give a ratio of 1.5 (2)\% in good agreement with older shake calculations of Byron and Joachain and of Åberg, as well as with recent many-body perturbation theory of Ishihara et al., calculations of Dalgarno and Sadeghpour, and of Andersson and Burgdörfer. In contrast to the intermediate energy behavior, consideration of final state correlations proves inessential as discussed by Dalgarno and Sadeghpour.
\end{abstract}

\section{Introduction}

Double photoionization of $\mathrm{He}$ has been used extensively as a testing ground for understanding correlation phenomena since $\mathrm{He}$ is the simplest atom which exhibits electron-electron interactions. Recently, there has been a great deal of progress experimentally and theoretically both near and far above threshold 
From the late seventies until the present, the threshold region has been extensively investigated $[1,2,3,4,5,6,7]$ and excellent agreement $[8,9]$ was found with Wannier theory [10]. More recently, in the high energy limit, measurements of the ratio of double-to-single photoionization at several photon energies, from 2 to $12 \mathrm{keV}$ have been reported to be 1.5 (2)\% [11], consistent with a calculated asymptotic value of $1.66 \%[12,13,14,15]$. Even though good agreement with this value of the ratio was obtained by older shake calculations $[12,13]$, a revised many body perturbation theory (MBPT) calculation [14], and with a procedure that uses the acceleration form of the dipole operator [15], it was only last year, after much debate over different and in part conflicting theories $[12,13,14,15,16]$, that an understanding of the relative importance of the different processes in double photoionization in the asymptotic limit was achieved. In particular, in this limit only ground state correlation need to be considered when using the acceleration gauge (cf. Dalgarno and Sadeghpour [15]). From about $2 \mathrm{keV}$ up, new theoretical studies of asymptotic behavior considering also the impact of Compton scattering by Andersson and Burgdörfer [17] have been made.

In contrast to the threshold and high energy regime, for intermediate energies few reliable theoretical values exist. Very recent theoretical calculations $[18,19]$ have investigated this range where the available data $[20,21]$ to test these differing theories are also extremely scarce, in fact previously nonexistent from $560 \mathrm{eV}$ to $2 \mathrm{keV}$. Further progress in understanding requires answering a leading question: what are the dominant correlation effects in the energy gap between threshold and below about $1500 \mathrm{eV}$, which force the ratio to undergo a significant decrease from about $5 \%$ to $2 \%$ before settling slowly into the asymptotic limit? Therefore, measurements in this intermediate energy range are of critical importance because they test the capability of the ab initio calculations to describe the transition between the low and high energy regime in an adequate way.

In this progress report, we present new measurements between $280 \mathrm{eV}$ and $1210 \mathrm{eV}$ and discuss recent measurements between $2 \mathrm{keV}$ and $12 \mathrm{keV}$ that test the most recent theories of Pan and Kelly [19], of Hino and Hino et al. [18] and of Andersson and Burgdörfer [17]. The aim of this work is to understand how the interplay of electron correlations, in both the initial and final states, affects the behavior of 
the ratio of double-to-single ionization, to understand the relative importance of the basis set, and of higher-order correlation effects.

\section{Experimental Techmique}

The intermediate energy measurements have been performed at the Berliner Elektronenspeicherring-Gesellschaft für Synchrotronstrahlung m.b.H. (BESSY). Monochromatic light from the high-energy toroidal grating monochromator beamline (HE-TGM-1) operated by the FritzHaber-Institute was tuned to several photon energies, hv, from $280 \mathrm{eV}$ to $1210 \mathrm{eV}$. The ions were analyzed by a TOF similar to the one used previously [22]. Several experimental effects can result in an inaccurate determination of the ratio of double-to-single ionization of helium and these have been discussed in previous work $[11,22,23]$. For this experiment, special emphasis has been put on suppressing possible higher-order and stray light contributions to the ion signal caused by the monochromator. In order to suppress the stray light effects, we took the measurements using a succession of filters to absorb unwanted low energy photons. The measurements were made just below the edge of each filter used. The higher-order light effects were taken into account by taking measurements just above the edge of the appropriate filter.

\section{Results and Discussion}

Our present ratios of double-to-single ionization obtained at several hv values in the intermediate energy range are shown in Fig. 1. They are compared with adjusted data of Bartlett et al. [21] corrected by them by a factor of 1.3 , in order to obtain agreement with earlier near threshold measurements. As can be seen from Fig. 1 our data agree quite well with the scaled data of Bartlett et al.

Our data are also compared in Fig. 1 with the recent MBPT calculations of Pan and Kelly [19] in the length (heavy short dashed line) and velocity form (heavy solid line) along with the recent MBPT calculations of Hino and Hino et al. [18] in the velocity (solid line) and the acceleration form (long dashed line) and with a semi-empirical calculation of Samson et al. [24] (chain line). Our data show excellent agreement with both the length and the velocity form of the calculations of Pan and Kelly, and 


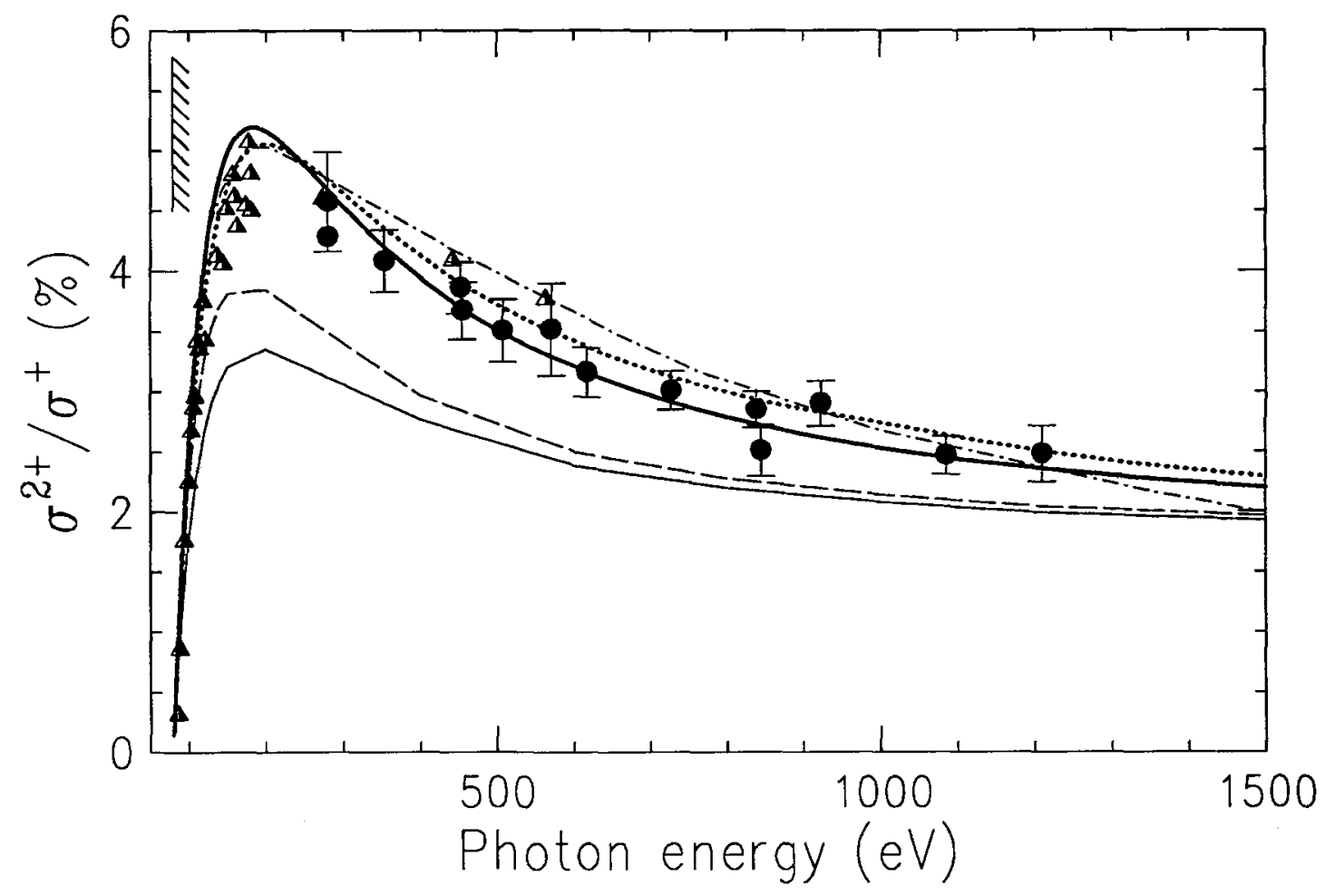

Fig. 1 Ratio of double-to-single ionization as a function of photon energy comparing the present data (full circles) with Bartlett et al. data (half filled triangles) [21], and with theories by Pan and Kelly (heavy solid and short dashed lines) [19], by Hino and Hino et al. (solid and long dashed lines) [18], and by Samson et al. (chain line) [24] (see text for details).

especially, with the velocity form. Pan has recently completed the calculation started by Pan and Kelly extending Carter and Kelly's threshold energy calculation [4] up to $14 \mathrm{keV}$ [19]. Throughout their calculation they included both ground state and final state correlations. As in the previous calculations [4], they show that while lowest order results show reasonable agreement with experiment, they found that certain higher-order correlation effects are significant. While agreement between the length and velocity forms is very good over this energy range, they find that separate total final state correlation (FSC) and ground state correlation (GSC) diagrams, while individually large and of nearly the same magnitude, are of opposite sign and therefore interfere strongly, with the FSC contribution being the larger of the two. The agreement with the data is therefore very good. The recent MBPT calculations of Hino and Hino et al. [18] using the velocity (the length form gives the same result as the velocity form) and the accelera- 
tion forms of the dipole operator lies lower than our data up to about $800 \mathrm{eV}$, with however, better agreement above that energy.

If indeed the main difference between Hino, Hino et al.'s MBPT calculation [18] and Pan and Kelly's MBPT calculation [19] is the inclusion of higher-order effects (both in GSC and FSC) and the use of a different basis set, (important since the choice of a pertinent basis set enables the implicit inclusion of higher-order effects), we are led to conclude that, at intermediate energies (below $1500 \mathrm{eV}$ ), unlike the high energy case, these higher-order effects are very important for the best description of the data. We also note that the acceleration form, which is less sensitive to higher-order effects, as used by Hino and Hino et al. [18] is not sufficient to describe the present data well.

Samson et al. [24] used a semi-empirical calculation based on a conjecture that there should be a proportionality between production of a doubly charged ion by photon impact on a neutral atom and electron impact on a singly charged ion. Electron impact data of Peart et al. [25] were scaled to obtain their curve. The chain curve, Fig 1, shows their calculation, which is in good agreement with the data. Overall good agreement is obtained in the present energy region, however, their model appears to break down above $1300 \mathrm{eV}$, since their curve decreases rapidly compared to the measured ratios at high energies $[11,22]$.

Figure 2 shows the intermediate energy data along with the high energy data [11]. They are compared with the recent calculations of Andersson and Burgdörfer [17], of Hino and Hino et al. [18] and of Pan and Kelly [19]. The MBPT calculations of Hino and Hino et al. [18] (solid for velocity and long dashed line for acceleration form) are low compared to the data below $2 \mathrm{keV}$. Hino [18] also calculates, using the acceleration form with an accurate ground state wave function and a correlated double continuum wave function for the final state, the values shown by the heavy chain line. His calculated values using this method are higher compared to the high energy data [11]. The calculated values by Andersson and Burgdörfer between $2-18 \mathrm{keV}$ which include contributions due to Compton scattering, agree very well with the high energy measurements. They use the acceleration form of the dipole opera- 


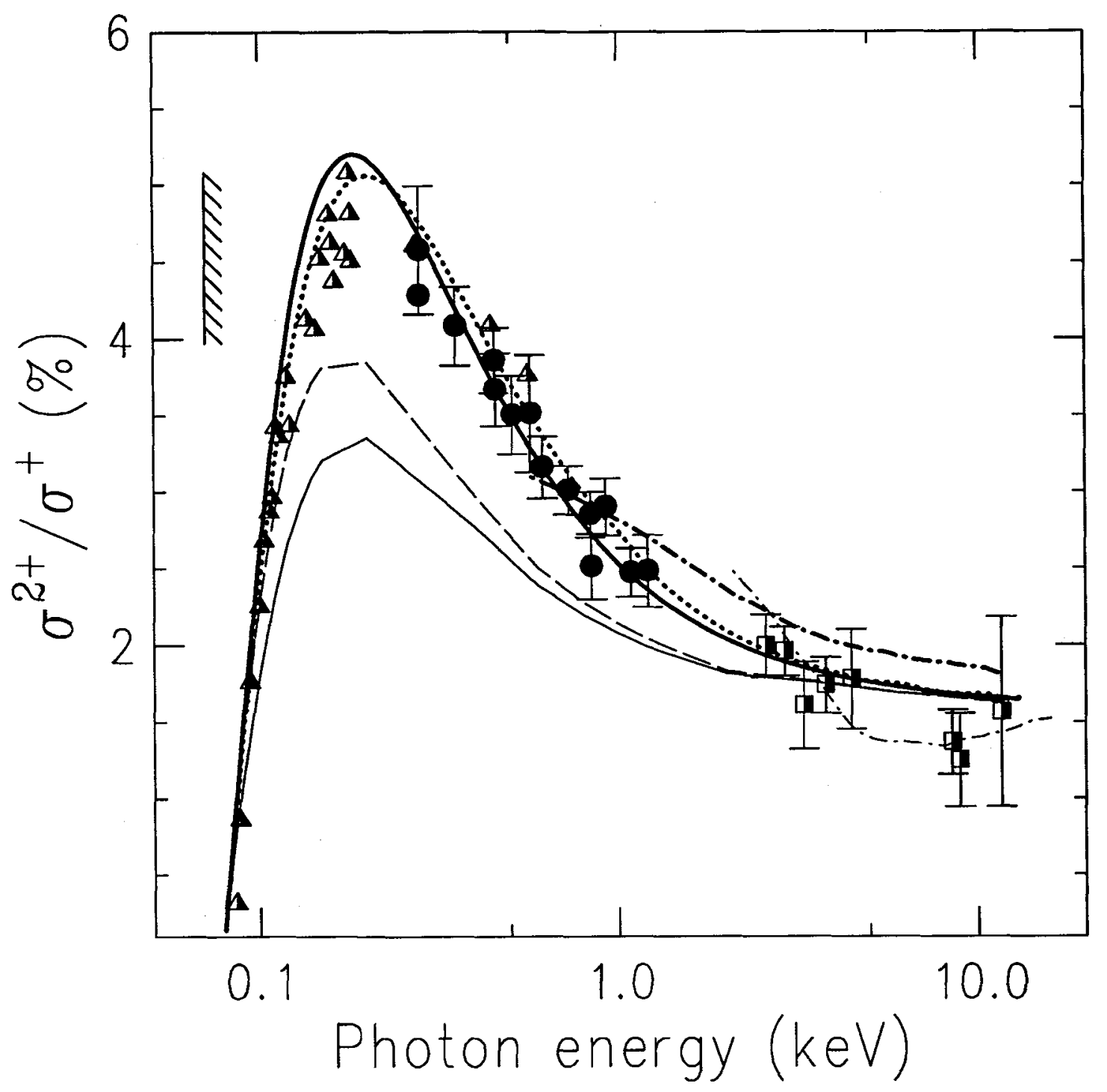

Fig. 2 Ratio of double-to-single ionization as a function of photon energy comparing the present data (full circles), the data of Levin et al. (half filled squares) [11] and the data of Bartlett et al. (half filled triangles) [21] with calculations by Hino and Hino et al. (solid, long dashed and heavy chain lines) [18], by Pan and Kelly (heavy solid and short dashed lines) [19], and by Andersson and Burgdörfer [17] (chain line) (see text for details).

tor and represent the electron-electron correlation in the final state by a Coulomb distortion factor. They avoid explicit representation of the two-electron continuum by summing ionization-excitation cross sections over all bound $\mathrm{He}^{+}$states to obtain the single-ionization cross section and by using sum rules. We can also see from Fig. 2 that the length and velocity form calculations of Pan and Kelly (as described 
above), which converge at about $2 \mathrm{keV}$, agree quite well with most of the high energy data even though Compton scattering effect is not included. This effect would tend to reduce Pan and Kelly's ratio , moving it in the direction of Andersson and Burgdörfer's value [17].

\section{Conclusions}

In summary, we have shown by comparing our measurements with the most recent calculations $[18,19]$ that at intermediate energies (below $1500 \mathrm{eV}$ ) both ground state and final state correlation appear to be important. At high energies, the measurements are consistent with an asymptotic value of $1.66 \%$ found with older shake calculation $[12,13]$ as well as with the most recent calculations $[14,15$, 17]. In particular, it was found [15] find that in this limit, only ground state correlation needs to be considered when using the accceleration gauge and that consideration of final state correlations is inessential.

Nora Berrah and Scott B. Whitfield are indebted to the Alexander von Humboldt foundation for fellowships. F. Heiser is indebted to the Deutscher Akademischer Austauschdienst for a PROCOPE fellowship. We are grateful to C. Pan and K. Hino for supplying their calculations prior to publication. This work was supported in part by the BMFT; by DoE, division of chemical science, under contract DE-FG02-92ER14299; by NIST; and by NSF.

\section{References:}

[1] V. Schmidt, N. Sandner, H. Kuntzemüller, P. Dhez, F. Wuilleumier, and E. Källne, Phys. Rev. A 13, 1748 (1976).

[2] G. R. Wight and M. J. Van der Wiel, J. Phys. B 9, 1319 (1976).

[3] D. M. P. Holland, K. Codling, J. B. West, and G. V. Marr, J. Phys. B 12, 2465 (1979).

[4] S. L. Carter and H. P. Kelly, Phys. Rev. A 24, 170 (1981).

[5] F. J. Wuilleumier, Ann. Phys. Fr. 4, 231 (1982). 
[6] R. Wehlitz, F. Heiser, O. Hemmers, B. Langer, A. Menzel, and U. Becker, Phys. Rev. Lett. 67, 3764 (1991).

[7] G. C. King, M. Zubek, P. M. Rutter, F. H. Read, A. A. MacDowell, J. B. West and D. M. Holland, J. Phys. B 21, L403 (1988); R. I. Hall, L. Avaldi, G. Dawber, M. Zubek, K. Ellis and G. C. King, J. Phys. B 24, 115 (1991); R. I. Hall, A. G. McConkey, L. Avaldi, K. Ellis, M. A. MacDonald, G. Dawber and G. C. King, J. Phys. B 25, 1195 (1992).

[8] H. Kossmann, V. Schmidt, and T. Andersen, Phys. Rev. Lett. 60, 1266 (1988).

[9] P. Lablanquie, K. Ito, P. Morin, I. Nenner, and J. H. D. Eland, Z. Phys. D 16, 77 (1990).

[10] G. H. Wannier, Phys. Rev. 90, 817 (1953).

[11] J. C. Levin, I. A. Sellin, B. M. Johnson, D. W. Lindle, R. D. Miller, N. Berrah, Y. Azuma, H. G. Berry, and D. -H. Lee, Phys. Rev. A 47, R16 (1993).

[12] F. W. Byron and C. J . Joachain, Phys. Rev. 164, 1 (1967).

[13] T. Åberg, Phys. Rev. A 2, 1726 (1970).

[14] T. Ishihara, K. Hino, and J. H. McGuire, Phys. Rev. A 44, R6980 (1991).

[15] A. Dalgarno and H. R. Sadeghpour, Phys. Rev. A 46, R3591 (1992).

[16] M. Ya. Amusia, E. G. Drukarev, V. G. Gorshkov, and M. P. Kazachkov, J. Phys. B. 8, 1248 (1975).

[17] L. R. Andersson and J. Burgdörfer, Phys. Rev. Lett. 71, 50 (1993).

[18] K. Hino, Phys. Rev. A 47, 4845 (1993); K. Hino, T. Ishihara, F. Shimizu, N. Toshima,, and J. H. McGuire, private communication, (submitted to Phys. Rev. A 1993). 
[19] C. Pan and H. P. Kelly, private communication (to be submitted to Phys. Rev. A 1993).

[20] T. A. Carlson, Phys. Rev. 156, 142 (1967).

[21] R. J. Bartlett, P. J. Walsh, Z. X. He, Y. Chung, E.-M. Lee, and J. A. R. Samson, Phys. Rev. A 46, 5574 (1992).

[22] J. C. Levin, D. W. Lindle, N. Keller, R. D. Miller, Y. Azuma, N. Berrah, H. G. Berry, and I. A. Sellin, Phys. Rev. Lett. 67, 968 (1991).

[23] N. Berrah, F. Heiser, R. Wehlitz, J. Levin, S. B. Whitfield, J. Viefhaus, I. A. Sellin, and U. Becker, Rapid Commu. PRA 48, R1733, (1993)

[24] J. A. R. Samson, R. J. Bartlett, and Z.X. He, Phys. Rev. A 46, 7277 (1992).

[25] B. Peart, D. S. Walton, and K. T. Dolder, J. Phys. B 2, 1347 (1969) 